Chirurg 2019 90 (Suppl 2):S27 https://doi.org/10.1007/s00104-019-0841-4 Online publiziert: 13. Februar 2019 (c) Springer Medizin Verlag $\mathrm{GmbH}$, ein Teil von Springer Nature 2019

\section{Originalpublikation}

Al-Batran SE, Hofheinz RD, Pauligk C et al (2016) Histopathological regression after neoadjuvant doxetacel, oxaliplatin, fluouracil, and leucoverin versus epirubicin, cisplatin, fluouracil, or capecitabine in patients with resectable gastric or gastroesophageal junction adenocarcinoma (FLOT4-AIO): results from the phase 2 part of a multicentre, openlabel, randomized phase $2 / 3$ trial. Lancet Oncol 17:1697-708

Hintergrund und Fragestellung. In der letzten Auflage der S3-Leitlinie „Magenkarzinom" ist die perioperative Chemotherapie als Standard für die lokal fortgeschrittenen Magenkarzinome und Karzinome des gastroösophagealen Übergangs Typ II und III definiert worden. Basierend auf der CunninghamStudie (Magic-Trial) gilt die perioperative Chemotherapie nach dem ECFSchema (Epirubicin, Cisplatin, 5-Fluoruracil) international als Therapie der Wahl. Zu der Frage der optimalen Chemotherapie wurde aktuell eine qualitativ hochwertige Studie (FLOT4-AIO) publiziert.

Methoden. In dieser prospektiv randomisierten Phase-2/3-Studien wurden in 28 deutschen onkologischen Zentren 300 Patienten mit einem lokal fortgeschrittenen Magenkarzinom oder Karzinom des gastroösophagealen Übergangs (AEG Typ I-III) eingeschlossen. Die Patienten

Dieser Beitrag wurde erstpubliziert in Der Chirurg (2017) 88:540-540. https://doi.org/10. 1007/s00104-017-0440-1

W. Schröder · C. J. Bruns

Klinik für Allgemein-, Viszeral- und Tumorchirurgie, Universitätsklinik Köln, Köln, Deutschland

\title{
Multimodale Therapie des Magenkarzinoms und Karzinoms des gastroösophagealen Übergangs - ECF vs. FLOT
}

wurden nach Randomisation entweder mittels ECF/ECX-Schema (152 Patienten mit jeweils 3 Zyklen prä- und postoperativ: Epirubicin, Cisplatin und 5-Fluoruracil/Capecitabine) oder dem FLOT-Regime (148 Patienten mit jeweils 4 Zyklen prä- und postoperativ: Doxecatel, Oxaliplatin, Leucoverin und 5-Fluoruracil) chemotherapiert. Aufgrund der inkludierten Karzinome reichte das chirurgische Spektrum von der subtotalen Gastrektomie bis zur transthorakalen Ösophagektomie. In der „Intention-totreat"-Analyse konnten insgesamt 137 Patienten in der ECF/ECX-Gruppe und 128 Patienten in der FLOT-Gruppe ausgewertet werden.

Ergebnisse. Als wesentliches Kurzzeitergebnis dieser Studie konnte gezeigt werden, dass die Rate der histopathologischen Vollremission in der FLOTGruppe signifikant höher war als bei den Patienten, die mit ECF/ECX therapiert wurden (16\%, $95 \%$-CI 10-23\% vs. $6 \%$, $95 \%$-CI 3-11\%; $p=0,02$ ). Bemerkenswert ist, dass in der FLOT-Gruppe eine signifikant höhere R0-Resektionsrate erzielt wurde ( $85 \%$ vs. $74 \%, p=$ 0,02). Weiterhin war die Gesamtrate der Patienten mit mindestens einer nichtchirurgischen oder chirurgischen schweren Komplikation (SAE, „serious adverse events") in der ECF/ECX-Gruppe signifikant höher als in der FLOT-Gruppe (44/111 Patienten, $40 \%$ vs. 30/119 Patienten, $25 \%$ ).

Fazit. Die vorliegenden onkologischen Daten dieser konzeptionell guten Studie zeigen in den Kurzzeitergebnissen eine nachweisliche Überlegenheit des FLOTRegimes in der Behandlung des Magenkarzinoms und der Übergangskarzinome und lassen erwarten, dass das Standardregime mittels ECF durch die FLOT-Therapie abgelöst wird. Hierfür müssen jedoch die noch nicht publizierten Langzeitergebnisse mit den 5-JahresÜberlebensraten abgewartet werden. Sollte sich hier auch die Überlegenheit der FLOT-Therapie nachweisen lassen, erscheint eine Anpassung der S3-Leitlinie „Magenkarzinom“ notwendig. Von besonderem Interesse ist auch die ausstehende Subgruppenanalyse der gastroösophagealen Karzinome Typ I, die gegenwärtig auch leitlinienkonform nach dem CROSS-Protokoll therapiert werden können.

\section{Korrespondenzadresse}

Prof. Dr. W. Schröder, FACS, FEBS

Klinik für Allgemein-, Viszeral- und

Tumorchirurgie, Universitätsklinik Köln Kerpener Str. 62, 50937 Köln, Deutschland wolfgang.schroeder@uni-koeln.de

Interessenkonflikt. W. Schröder und C. J. Bruns geben an, dass kein Interessenkonflikt besteht. 